\title{
Palomar Testbed Interferometer
}

\section{J. Kent Wallace, Andrew F. Boden, M. Mark Colavita, Philip J. Dumont, Yekta Gursel, et al.}

J. Kent Wallace, Andrew F. Boden, M. Mark Colavita, Philip J. Dumont, Yekta Gursel, Braden E. Hines, Christopher D. Koresko, Shrinivas R. Kulkarni, Benjamin F. Lane, Fabien Malbet, Dean L. Palmer, Xiaopei Pan, Michael Shao, Gautam Vasisht, Gerard Theodore van Belle, Jeffrey W. Yu, "Palomar Testbed Interferometer," Proc. SPIE 3350, Astronomical Interferometry, (24 July 1998); doi: 10.1117/12.317154

Event: Astronomical Telescopes and Instrumentation, 1998, Kona, HI, United States 


\title{
Palomar Testbed Interferometer
}

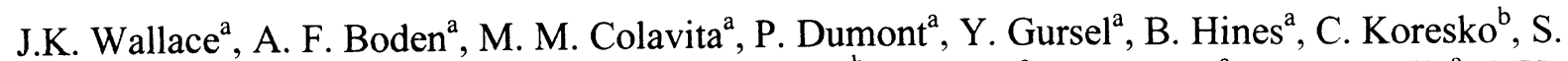 \\ Kulkarni ${ }^{\mathrm{b}}$, B. Lane ${ }^{\mathrm{a}}$, F. Malbet ${ }^{\mathrm{c}}$, D. Palmer ${ }^{\mathrm{a}}$, X.P. Pan ${ }^{\mathrm{b}}$, M. Shao ${ }^{\mathrm{a}}$, G. Vasisht ${ }^{\mathrm{a}}$, G. van Belle ${ }^{\mathrm{a}}$, J. Yu $^{\mathrm{a}}$ \\ a Jet Propulsion Laboratory, California Institute of Technology, \\ 4800 Oak Grove Dr., Pasadena, CA 91109 \\ ${ }^{\mathrm{b}}$ California Institute of Technology \\ ${ }^{\mathrm{c}}$ LAOG, Observatoire de Grenoble, France
}

\begin{abstract}
The Palomar Testbed Interferometer (PTI) is an infrared, phase-tracking interferometer in operation at Palomar Mountain since July 1995. It was funded by NASA for the purpose of developing techniques and methodologies for doing narrowangle astrometry for the purpose of detecting extrasolar planets. The instrument employs active fringe tracking in the infrared $(2.0-2.4 \mu \mathrm{m})$ to monitor fringe phase. It is a dual-star interferometer; it is able to measure fringes on two separate stars simultaneously. An end-to-end heterodyne laser metrology system is used to monitor the optical path length of the starlight. Recently completed engineering upgrades have improved the initial instrument performance. These upgrades are: extended wavelength coverage, a single mode fiber for spatial filtering, vacuum pipes to relay the beams, accelerometers on the siderostat mirrors and a new baseline. Results of recent astrometry data indicate the instrument is approaching the astrometric limit as set by the atmosphere.
\end{abstract}

Keywords: optical interferometry, astrometry, extrasolar planets

\section{INTRODUCTION}

The Palomar Testbed Interferometer ${ }^{1}$ is an infrared interferometer located at Palomar Mountain, California. It has a $110 \mathrm{~m}$ baseline oriented in a nominal North-South direction. The apertures are $40 \mathrm{~cm}$; this is roughly equal to $r_{0}$ at $2.2 \mu \mathrm{m}$ under typical atmospheric conditions at the site. The fringes are actively tracked with delay line trolleys that have an optical delay range of $+/-38.3 \mathrm{~m}$. A singe line of a NICMOS III array is used for fringe detection. The broad band (or white) light and low resolution spectrometer are imaged onto a common row of the array and are read out simultaneously. Custom clocking of this array results in a final read noise of about $15 \mathrm{e}-$. Angle tracking is done at a center wavelength of $0.8 \mu \mathrm{m}$ with a quad cell implemented as a lenslet array. The lenslet couples light into four fibers, each of which is coupled to a photon-counting avalanche photodiodes. The instrument control is accomplished via seven single-board real-time computers. These do the sequencing and automation of the instrument. This paper will review the instrument, discuss some of the recent modifications, describe the details of the astrometry measurement and show some recent results.

\section{DESCRIPTION OF THE INTERFEROMETER}

The instrument will be described in the following section. The description will begin at the siderostats and finish at the fringe tracker. Each sub-system along the beam train of the star light path will be described in detail as it is encountered.

A schematic of the pier is shown in Fig. 1. The siderostats are the first optic in the train. The mirrors are $50 \mathrm{~cm} Z$ Zerodur flats; they weigh over $90 \mathrm{lbs}$. The mounts are alt-az gimbals and are designed such that the mirror surface and the intersection of the bearings are coincident. The siderostats reflect the starlight into the fixed direction of a telescope. This $40 \mathrm{~cm}$ diameter $\mathrm{f} / 10$ Cassegrain telescope is mounted on an optical 4'x8' optical table tilted downwards by fifteen degrees. The telescope is mounted in an athermal structure so as to minimize re-focusing as thermal conditions change. 


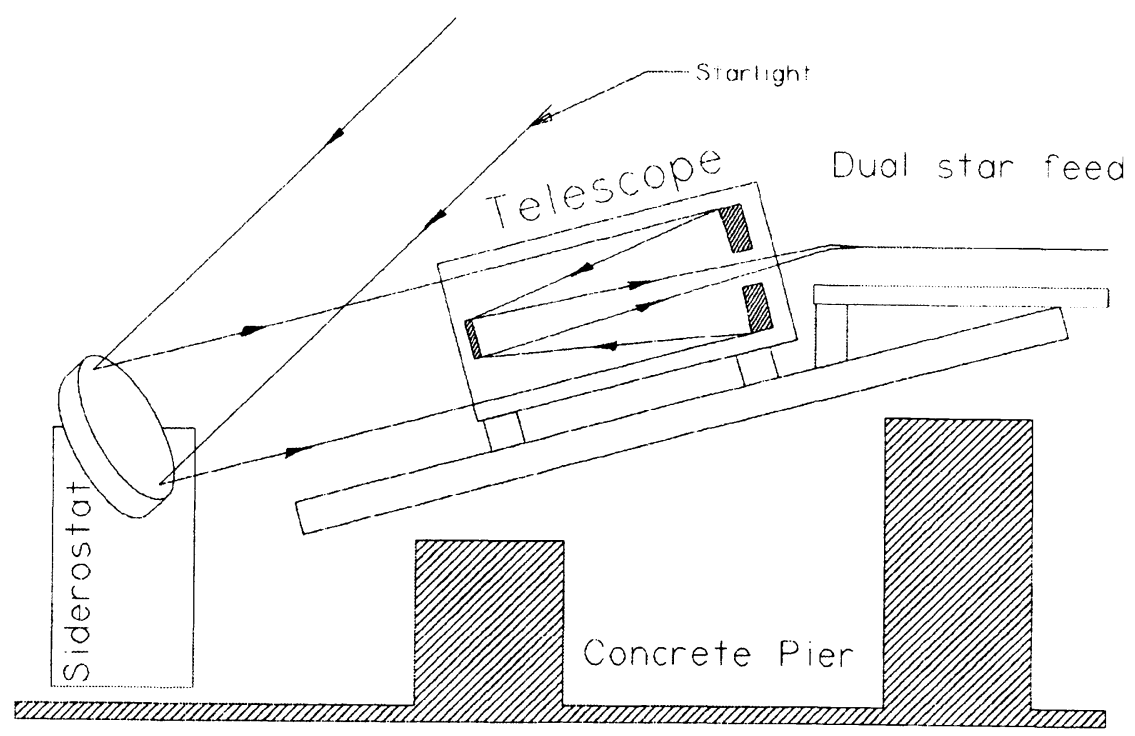

Figure 1

Mounted between the siderostat and telescope, in the shadow of the secondary mirror is a pick-off mirror which folds this part of the incoming starlight down and towards the side of the telescope. A similar mirror on the table directs the light to a 3.5" diameter Questar (C) telescope mounted parallel to the Cassegrain on the optical table. The incoming starlight is brought to a focus on the acquistion camera.

A corner cube mounted near the first fold mirror picks off part of the light from a laser boresight. This boresight light threads its way from the main beam combining tables at the heart of the instrument out to the telescope. The fraction of this light picked off is turned around and displaced such that it follows the path of the star light into the acquisition camera. The instrument boresight and the starlight are thus clearly visible on the wide-angle acquisition camera. When the siderostat is adjusted such that the images are coincident, the starlight is boresighted to the interferometer.

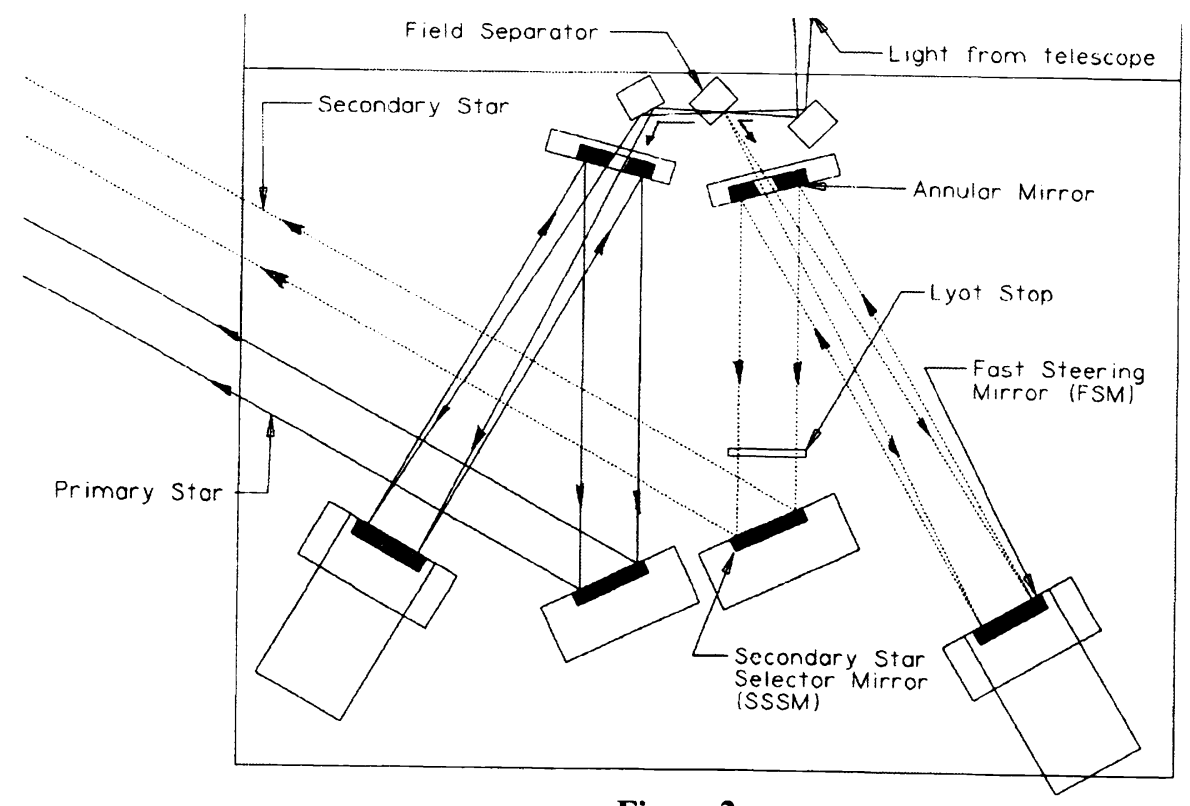

Figure 2 
Fig. 2 is a schematic of the dual-star feed; it is useful in the following discussion. The starlight leaving the telescope is directed upwards to a horizontally mounted 3' $\mathrm{4}$ ' optical breadboard. This is the dual-star feed; it's where the two stars are separated. A single fold brings this light level with the breadboard. In the focal plane is a field separator (currently a beamsplitter) which separates the light from the bright, nearby (or primary) star and the faint science (or secondary) star. Each beam passes through an annular mirror to an on axis parabola where they are re-collimated with a diameter of $7.5 \mathrm{~cm}$. These parabolas are also fast steering mirrors. The mirrors are in friction-free flexure mounts and are tip/tilt controlled by PZT's. They receive feed back from the angle tracker located on the main beam combining table, near the end of the optical train.

From the parabola the starlight travels back to the annular mirror which directs the light to a steering mirror. From here, the light is directed to the main building. For the secondary beam path, the steering mirror is an image of the siderostat mirror. Its task is to select the secondary star. Since it is an image of the entrance pupil, the secondary star selection is done without introducing pupil sheer into the system.

At the main building, the beams cross the wall of the structure at the crook on the underside of the ' $T$ '. The beams are then directed down towards the long end the delay lines. Here the beams are delayed so they are phased when they reach the main beam splitter on the beam combiner tables. The beams come in 'high' or 10" above the switchyard table, and after passing through the cat's eye mirror of the delay line, they are 6" above the switchyard table (thus missing the input mirrors on the return path).

The delay lines ${ }^{3}$ slave together the primary and secondary starlight from a single pier and delay them in unison. The active delay line is implemented as a cat's eye trolley with a four-stage servo system. It has a range of $+/-38$ meters with a resolution of a few nanometers. A passive delay line allows for very coarse positioning. Once the passive is located, the difference in delay positions is tracked out with the active delay line. The location of each trolley is monitored with its own heterodyne laser metrology system.

The beams next pass through a pair of confocal parabolas which constitute the beam compressors. These take the beam from a diameter of $75 \mathrm{~mm}$ down to $20 \mathrm{~mm}$. The secondary beam is folded to its beam combiner table. The primary beam goes to a pair of cat's eye retro-reflectors, one of which is on a translation stage. It is this moveable cat's eye which is the differential delay line between the primary and secondary tables. After it passes through this set of optics, the primary beam is directed towards its beam-combining table.

Figure 3 shows a schematic of the primary beam combiner table. This table contains the star-tracker, fringe tracker, whitelight source and boresight laser. A 6 " diameter dichroic mirror reflects starlight short of $1 \mu \mathrm{m}$ to the star-tracker where it is

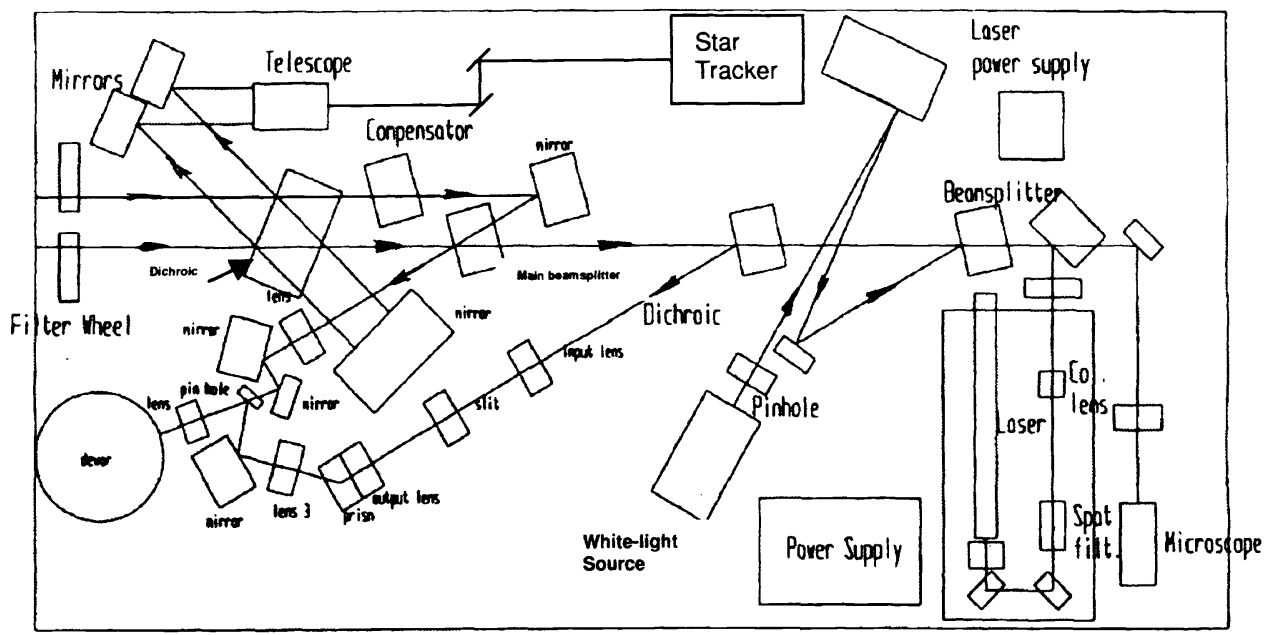

Figure 3 
sensed for tilt. Each beam combiner has a single quad cell for tilt sensing which is shared between the beams from the two piers. This quad cell is implemented as a lenslet array feeding four multimode fibers which are each coupled to a photon counting avalanche photodiode. The starlight from each pier is chopped onto the quad cell every $10 \mathrm{msec}$ for a duration of 5 msec. A PZT mirror on which the beams overlap chops the spots back and forth across the quad cell. The optical bandwidth of the star tracker is $0.7 \mu \mathrm{m}$ to $1.0 \mu \mathrm{m}$. The lower edge is set by the filters that block the stray laser metrology light, while the upper end is set by the response of the APD. The sample rate is $100 \mathrm{~Hz}$ and the closed-loop bandwidth of the star tracker is $10 \mathrm{~Hz}$. The limiting magnitude is fainter than $10 \mathrm{~m}_{\mathrm{R}}$

The IR light proceeds to the main beam splitter. Light from the 'front' side of the beam splitter is directed via relay optics to a single pixel of the IR array located inside the dewar. The light from the other side of the beam splitter encounters a dichroic which transmits the visible light while reflecting the IR light. The light then passes through a prism for low dispersion. After a fold mirror and another lens, the light is coaligned with the white light arm via a reflecting half-plane. This spectrometer light is re-imaged on the same line of the array which contains the white light.

A white light source is used for calibration of the pixels on the spectrometer as well as for diagnostics of the instrument. By placing corner cubes after the long delay lines, and using the white light source, it is possible to observe internal white-light fringes which is useful for performing engineering tests. A narrow band filter placed in the white light path is used to calibrate the pixels in the spectrometer. The boresight laser defines the optical axis of the instrument. The beam is collimated and follows the reverse path of the stellar light through the instrument from the main beam combiner out to the dual star feed optics at the piers and through the telescope. The laser ties together the white light source, the angle tracker and the acquisition system in a nightly calibration procedure. The microscope at the end of the table is used for visual alignment and for other instrument diagnostics.

A laser metrology system is used to measure the full optical path of the system from where the beams are combined at the main beamsplitter out to a fiducial corner cube located on the dual-star feed. Since both the primary and secondary star metrology paths are common to this corner cube, there is the possibility that the signals may become mixed. Therefore, the beams are uniquely identified in two ways. First, the beat frequencies are distinct for each table; the primary has a frequency of $440 \mathrm{kHz}$ and the secondary has one of $110 \mathrm{kHz}$. Each detector has a corresponding pass band filter matched to its frequency. There is an offset for the above signals is on the order of $30 \mathrm{Mhz}$. This prevents false signals due to leakage since the inter-table frequency difference is well beyond the bandwidth of the detectors. Also, the polarization state coming from each table is orthogonal to the polarization of the other table at the pier. This prevents mixing of the two beams at the detectors.

The instrument is controlled with 7 real-time computers in three VME crates. Each sub-system is controlled by a singleboard 68040 computer running real time software developed under the VxWorks operating system. Each CPU communicates with others via reflective memory. A graphical user interface (GUI) that resides on a local Sun workstation controls the realtime system. The Sun is also used for data management. A typical observing procedure involves the operator creating an ordered list of objects to observe. This list is loaded into the GUI at the beginning of the evening. The instrument is highly automated and very efficient; it is possible to observe greater than 100 different interferometric scans per night. A typical observing sequence is illustrated below in Fig. 4.

When a new star observation is requested, the delay lines and siderostats slew to their nominal location. The acquisition camera images the star and feeds offsets to the siderostats to boresight the starlight with the interferometer. The star will then be within the narrow field of view of the star trackers. Once the star is being tracked, a couple of calibration measurements are made. First, the delay line is offset to a point of no interference. The total flux coming from both apertures is measured for thirty seconds. One of the beams is then blocked and another thirty seconds of data is taken from a single aperture. Next, the fringes are tracked for two minutes. After the fringes have been observed, the siderostats off-point by a small amount and measure the contribution of the background. At this point, the interferometer is ready for a new star. This process takes a total of six to eight minutes depending upon observing conditions. 


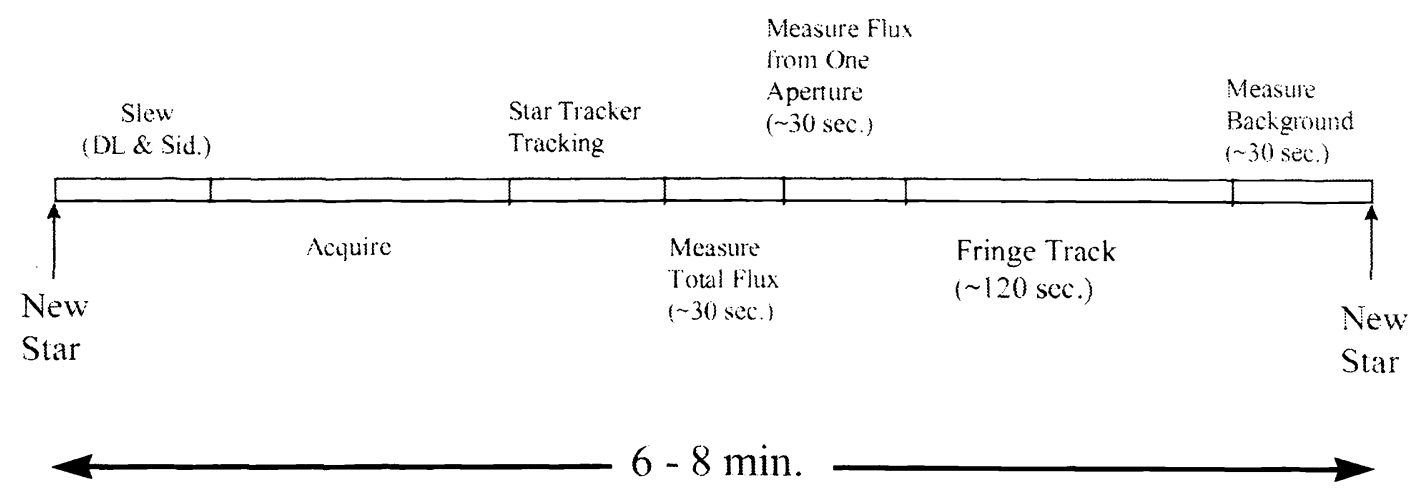

Figure 4

\section{RECENT UPGRADES TO THE INSTRUMENT}

Several improvements have to made to the basic instrument to increase it capabilities and to enhance its performance. All of these improvements have been completed in 1997. The goal of most of these improvements has been to enhance the astrometry measurement by either improving the system visibility or by reducing the extent of phase fluctuations.

\subsection{Extended wavelength coverage to $H$ band.}

All of the optics up to the main beam combining tables are reflective (save for the IR fused silica windows which cap both ends of the beam transfer tubes). Therefore, extending the wavelength range for the system amounted to replacing the main starlight beamsplitter as well as the coupler in the spectrometer arm. Likewise, the lenses that couple light into the dewar were replaced with new IR achromats to provided diffraction limited performance over the $\mathrm{H}$ and $\mathrm{K}$ bands $(1.5-2.5 \mu \mathrm{m})$. $\mathrm{A}$ custom $\mathrm{H} / \mathrm{K}^{\prime}$ astronomical filter inside the dewar housing the NICMOS 3array completed the modification.

\subsection{Single-mode IR optical fiber in Spectrometer Arm}

An infrared optical fiber (Le Verre Fluoré, France) was incorporated into the low-resolution spectrometer arm of the beam combiner table in order to spatially filter the wave front. Aberrations in the wave front (due to both static, or slowly varying wave front degredation due to the instrument, as well as quickly changing atmospheric effects) result in a reduction of fringe visibility. The fiber removes the photons which do not contribute to the interference and which would otherwise be detrimental to the visibility. Light is coupled in-to and out-of the fibers using off-axis portions of full parabolic reflectors. These reflectors are on kinematic bases, so they can be removed from the nominal beam path and replaced in a repeatable fashion. The effect of using the fibers has been to improve the raw $\mathrm{V}^{2}$ for unresolved objects by a factor of two (from $\sim 0.4$ to 0.9). Science projects involving parametric modeling have benefited most from this improvement, since they rely soley on the spectrometer channel for visibility data.

\subsection{Vacuum pipes}

In their original incarnation, the light paths going from the piers to the main building where enclosed in 6" diameter aluminum pipes which where also wrapped in foil-faced bubble-wrap and then finally covered with white PVC jacketing for protection against the elements. The ends of the pipes where sealed with 5" diameter IR fused silica (Infrasil 301, Heraeus). These pipes transported the beams to minimize the effects due to turbulence, which it would otherwise see over a long propagation distance in the air, as well as at the interface between the main building and the outside. Although, in steady state conditions the air pipes were sufficient, during the transition from day-to-night the pipes were still susceptible to the effects of residual turbulence. It's thought that, although care was taken to insulate the pipes, at night the tops of the air pipes radiatively cooled to the sky, while the bottom of the pipes were exposed to the warm ground. It is this temperature differential which was responsible for setting up the convective cells.

The four sets of 6" aluminum pipes have been replaced with 6" steel vacuum pipes which span the distance between the main building and the piers. These pipes are pumped down with a portable roughing pump located inside the main building. Once pumped down, they hold vacuum sufficiently well so as not be pumped again for a few weeks. 


\subsection{Siderostat Accelerometers}

The system has operated for some time with a residual amount of OPD piston jitter attributable to the siderostat. Although the optical path length is monitored with laser metrology, this metrology does not extend to the siderostats themselves. This motion is off-loaded to the delay lines by the fringe tracker. This is a rather indirect way of monitoring the piston jitter.

The siderostats are now included as part of the fringe phase control via a more direct means. Accelerometers have been added to the back of the siderostat mirrors. These accelerometers measure the motion normal to the mirror's front surface above the $\sim 1 \mathrm{~Hz}$ level. The component of this vibration along the boresight of the instrument is then fed forward (in an open loop) to the active delay line where this additional path modulation is removed. The addition of the accelerometers has resulted in the reduction of the phase noise at the resonance frequency by $10 \mathrm{~dB}$.

\subsection{New N-W baseline}

When the facility was constructed, a third pier was added for the potential of a future expansion. Likewise, a third siderostat mount and mirror were purchased with this same possibility in mind. Therefore, adding the third baseline involved only a few procurements and was relatively easy to incorporate into the already working instrument. Fringes were obtained on this baseline at the end of last years observing season. The new baseline is roughly orthogonal to the current baseline.

\section{ASTROMETRY WITH PTI}

\subsection{Overview of the astrometric measurement}

Validating techniques for narrow-angle astrometry is one of the key reasons why PTI was constructed ${ }^{2}$. What follows is a brief description of the astrometric measurement.

The dual-star feed enables the interferometer to track fringes on two different stars simultaneously. By measuring both the difference in delay ( $\mathrm{d}$ in Fig. 5a) for fringes and the fringe phase, it's possible to determine the angular separation of the stars (assuming the baseline is known). The process is as follows: first fringes are obtained on the primary fringe tracker. Once the primary has acquired fringes it continues in this mode for the entire observation. The secondary delay line is adjusted until it too gets fringes on the same object. It takes fringe data in this mode for a while. The secondary channel then points to

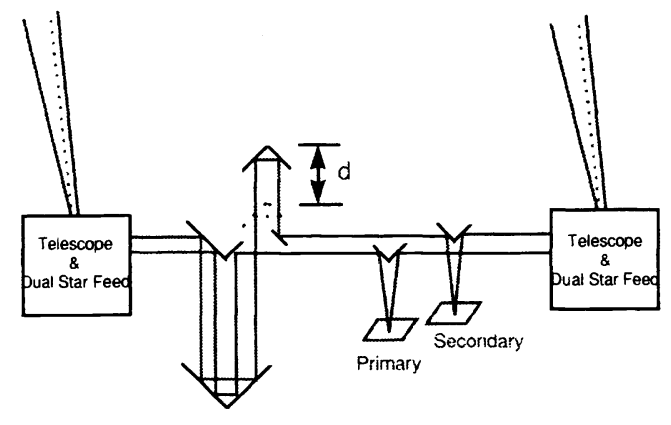

Figure 5a
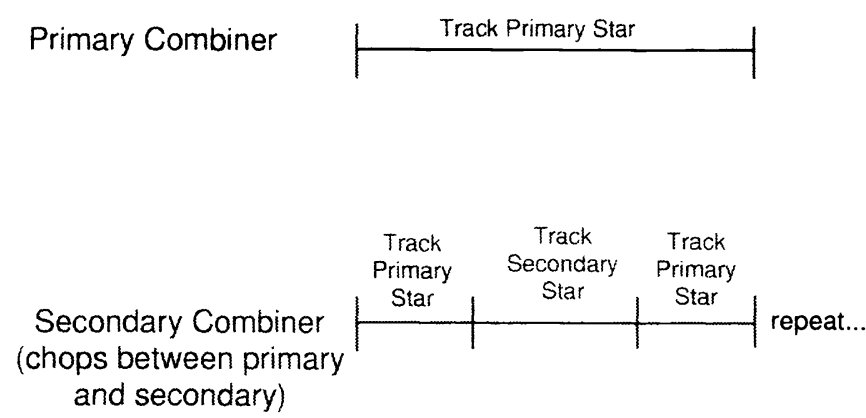

Figure 5b

its star and its delay line is adjusted until fringes are observed on the secondary object. After tracking fringes on the secondary object for a while, the secondary channel re-points and the delay lines re-phase to the primary object. The measurement of the secondary object is thus bracketed by observations of the primary object in order to take out instrumental drifts which may have occurred during the secondary observation. This technique of looking alternately between a primary and secondary object and then back to the primary object "chopping". It is both the difference in delay line position (which is measured by the constant term metrology) between the primary and secondary objects and the phases of both fringes which are the measurables. An illustration of the observing sequence is shown in Figures 5a and 5b. 


\subsection{Recent Astrometry Results}

Figure 6 below shows data from an astrometric measurement done at PTI. There are several aspects of the data which are worth noting. First it should be pointed out that the gaps are due to loss. The upper curve is the delay for the primary star as it moves across the sky. The bottom curve is the differential delay between the primary and secondary stars magnified by a factor of 10. The differential delay is roughly $15 \mathrm{~mm}$. With PTI's baseline of $110 \mathrm{~m}$, the angular separation between the stars is given by $15 \mathrm{~mm} / 110 \mathrm{~m}=30 \mathrm{arc} \mathrm{sec}$. The differential delay data also arcs due to the sidereal motion. From the delay as a function of time, it's possible to determine the trajector of the secondary object.

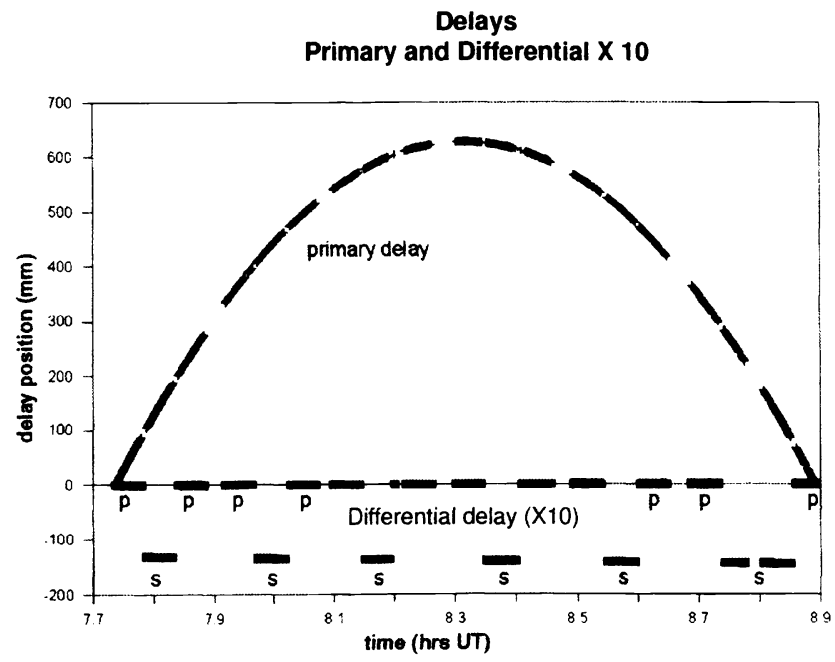

Figure 6

Data from another night is shown below in Fig. 7. Each data point represents a 0.5 second average; each group of data points is roughly 380 seconds long. As mentioned previously, data is taken on the primary star both before and after the observation of the secondary object. A linear model is fit to this data which is the instrument bias during the secondary observation. The upper section of Fig. 7 is the calibrated difference between the primary and secondary delay. This data is then fit for the sidereal motion. The residual after this fit is the second section of the plot. The bottom plot shows the average of each 380 second scan. The RMS value of these points is roughly 0.7 mas. If we assume white noise in the errors, the error scales to 220 uas after one hour of intergration.

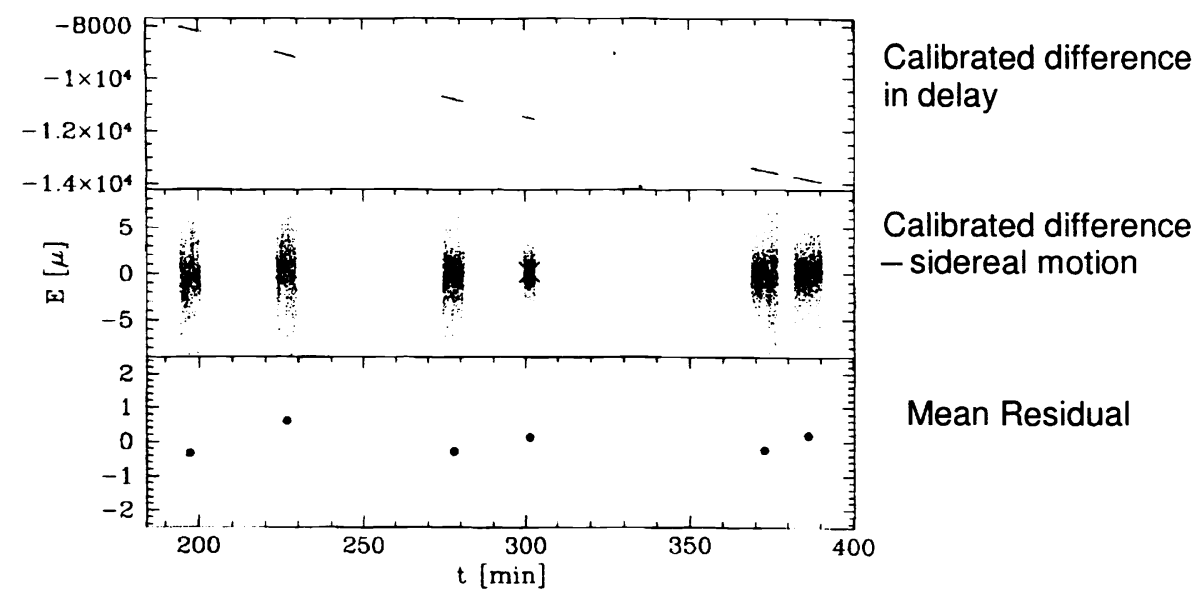

Figure 7 


\section{ACKNOWLEDGMENTS}

Funding for the development of PTI was provided by NASA under its TOPS (Towards Other Planetary Systems) and ASEPS (Astronomical Studies of Extrasolar Planetary Systems) programs, and from the JPL Director's Discretionary Fund. Ongoing funding has been provided by NASA through its Origins Program and from the JPL Directors Research and Development Fund. Thanks to Kadri Vural and Rockwell International for the NICMOS-3 detectors.

The work reported here was performed at the Jet Propulsion Laboratory, California Institute of Technology, under a contract with the National Aeronautics and Space Administration.

\section{REFERENCES}

1 M.M. Colavita, M. Shao, B.E. Hines, J.K. Wallace, Y. Gursel, F. Malbet, J.W. Yu, H.Singh, C.A. Beichman, X.P.Pan, T. Nakajima, S.R. Kulkarni, “ASEPS-0 Testbed Interferometer," Proc. SPIE 2200, 89-97 (1994).

2 M. Shao and M.M. Colavita, "Potential of long-baseline infrared interferometry for narrow-angle astrometry," Astron. Astrophys. 262, 353-358 (1992).

3 M.M. Colavita, B.E. Hines, M. Shao, “A high speed optical delay line for stellar interferometry," in High Resolution Imaging by Interferometry II, ESO Conf. and Workshop Proc. 39, 1143-1147 (1992).

Further information: http://huey.jpl.nasa.gov/palomar. 